\title{
ON A THEOREM OF VON NEUMANN
}

\section{DOROTHY MAHARAM}

1. Introduction. Let $S$ be a measure space with a countably additive, $\sigma$-finite, complete (non-negative) measure. J. von Neumann has proved that, from each class $x$ of measurable sets modulo null sets of $S$, a representative set $R(x) \in x$ can be picked in such a way that $R(x \vee y)=R(x) \cup R(y), \quad R(x \wedge y)=R(x) \cap R(y), \quad$ and $\quad R(-x)$ $=S-R(x)$. That is, the correspondence $x \rightleftarrows R(x)$ is a finite Boolean isomorphism between the algebra of measurable sets modulo null sets of $S$, and some subalgebra of the algebra of measurable sets of $S$. He first proved this theorem for the case in which $S$ is the real line ${ }^{1}$, and later (around 1942) gave an oral proof of the general theorem to S. Kakutani and the author. This proof was unfortunately forgotten beyond hope of reconstruction; a proof (which the author believes to be different) is supplied here.

The problem of selecting representatives from the measure classes is, in a sense, equivalent to that of defining upper and lower density functions $D^{*}, D_{*}$, (with the expected properties) for the measurable sets. In fact, if the representatives are known, we can trivially define $D^{*}{ }_{X}=D_{* X}=$ characteristic function of $R(x)$ for each set $X \in x$, while conversely, as von Neumann has shown in [4], if $D^{*}$ and $D_{*}$ are known (and are not affected, of course, when $X$ is altered by a null set), we may choose a suitable $R(x)$ between the "upper density set" of $X$ (where $D^{*}{ }_{X}$ is positive) and the "lower density set" (where $D_{* X}$ is 1), by a skillful application of the axiom of choice. Further, by [2], the case of a general measure space can be reduced, with a little trouble, to that of a product of (in general, uncountably many) unit intervals $I_{\lambda}$. Thus the problem is transformed to that of defining suitable density sets or functions in a product space ${ }^{2} \prod I_{\lambda}, \lambda \in \Lambda$. Unfortunately the straightforward approach by directed limits does not work here, as J. Dieudonné has shown in [1]. We shall therefore suppose the factors to have been well-ordered (so that $\lambda$ runs over a section of ordinals), and show that a quite natural procedure (involving no further use of the axiom of choice) leads to suitable density

Presented to the Society February 23, 1957; received by the editors May 2, 1958.

${ }^{1}$ See [4] and [5]. If the continuum hypothesis is assumed, a simple transfinite induction suffices.

${ }^{2}$ We actually consider, in the first instance, not $\prod I_{\lambda}$ but a product of 2-point factors $Q_{\lambda}$, each point having weight $1 / 2$. The details for $\prod Q_{\lambda}$ are easier, and the theory for $\prod I_{\lambda}$ is derived from it later. 
sets; the axiom of choice is needed again to pick the required representatives. The same procedure also defines well-behaved density functions $D^{*}, D_{*}$, in $\prod I_{\lambda}$, without the axiom of choice; but they are rather more artificial.

One by-product of the investigation deserves notice: it turns out that, in a product space such as $I I I_{\lambda}$, every open set is measurable.

2. Density sets in products of 2-point spaces. Let $\left\{Q_{\alpha}\right\}\left(\alpha<\alpha^{*}\right)$ be a well-ordered family of 2 -point spaces $Q_{\alpha}$, each consisting of two points $a_{\alpha}, b_{\alpha}$ each of measure $1 / 2$; the suffix $\alpha$ runs over all ordinals less than some (infinite) ordinal $\alpha^{*}$. The product space $Q=\prod Q_{\alpha}$ is made into a (complete) measure space in the usual way, the field of measurable sets being generated, modulo the null sets, by the "elementary" sets which can here be described as follows. For any finite set of suffixes $\alpha_{1}, \alpha_{2}, \cdots, \alpha_{n}$, choose a point $r \in \prod\left\{Q_{\alpha_{i}} \mid i=1,2, \cdots, n\right\}$; then the set $(r) \times \prod\left\{Q_{\alpha} \mid \alpha<\alpha^{*}, \alpha \neq \alpha_{1}, \alpha_{2}, \cdots, \alpha_{n}\right\}$ is a typical elementary set.

Theorem 1. We can define, for each measurable subset $X$ of $Q, a$ measurable set $\theta(X) \subset Q$, in such a way that ${ }^{3}$ :

(i) If $X \subset Y, \theta(X) \subset \theta(Y)$;

(ii) $\theta(X \cap Y)=\theta(X) \cap \theta(Y)$;

(iii) If $X$ and $Y$ differ by a null set, $\theta(X)=\theta(Y)$;

(iv) $\theta(X)$ differs from $X$ by a null set at most;

(v) If $F$ is a finite union of elementary $\operatorname{set}^{4}, \theta(F)=F$.

Before proving the theorem, we remark that these properties (except for $(v))$ are familiar properties of the lower density set on the line. An upper density set is obtained by taking $Q-\theta(Q-X)$; this contains $\theta(X)$, from (iii) and (v), and has the dual properties. To obtain a lower density function, we have only to take $D_{* X}=$ characteristic function of $\theta(X)$; this has all the expected properties (that corresponding to (ii) being $1+D_{* X \cap Y} \geqq D_{* X}+D_{* Y}$ ), while conversely if $D_{*}$ is given and has the properties corresponding to (i)-(v), and we take $\theta(X)=$ set where $D_{* X}=1, \theta$ satisfies the requirements of the theorem. Similar remarks apply to the upper density set and function.

Proof of Theorem 1. For each ordinal $\beta \leqq \alpha^{*}$ (but $>1$ ) we write $R_{\beta}=\prod\left\{Q_{\alpha} \mid \alpha<\beta\right\}$ and, if $\beta<\alpha^{*}, S_{\beta}=\prod\left\{Q_{\alpha} \mid \beta \leqq \alpha<\alpha^{*}\right\}$; thus $Q$ $=R_{\beta} \times S_{\beta}\left(1<\beta<\alpha^{*}\right)$. The natural projection from $Q$ to $R_{\beta}$ is denoted by $\pi_{\beta}$. A set of the form $\pi_{\beta}^{-1}(Y)$, where $Y$ is a measurable subset of

${ }^{3}$ As the referee points out, postulate (i) is redundant, being a consequence of (ii).

${ }^{4}$ In particular, $\theta(F)=F$ when $F=\phi$ or $Q$. ( $\phi$ denotes the empty set; its measure class is denoted by 0 .) 
$R_{\beta}$, is called a "cylinder set on $R_{\beta}$ "; if $\beta<\alpha^{*}$ it is of the form $Y \times S_{\beta}$. Similarly a function $f$ on $Q$ which is of the form $f(q)=g\left(\pi_{\beta}(q)\right)$, where $g$ is a measurable function on $R_{\beta}$, is a "cylinder function on $R_{\beta}$ "; we have (if $\left.\beta<\alpha^{*}\right) f(r, s)=g(r)$ where $r \in R_{\beta}, s \in S_{\beta}$. The mapping $\pi_{\beta}$ induces a measure-preserving isomorphism between the Boolean algebras of cylinder sets on $R_{\beta}$ on the one hand, and of measurable subsets of $R_{\beta}$ on the other.

We shall define $\theta(X)$ by transfinite induction, defining it successively for the cylinder sets on $R_{\beta}, \beta=2,3, \cdots$; when $\beta=\alpha^{*}$, the theorem is proved. For finite values of $\beta$ the situation is trivial $\left(R_{\beta}\right.$ is finite and atomic, $\theta(X)=X$ for each cylinder set on $R_{\beta}$ ). Suppose, then, that $\beta$ is infinite and $\leqq \alpha^{*}$, and that $\theta(X)$ has been defined for all cylinder sets on $R_{\alpha}$, for all $\alpha<\beta$, and has the properties stated in the theorem so far as they apply (i.e., when $X, Y, F$ are restricted to be cylinder sets on $\left.R_{\alpha}, \alpha<\beta\right)$ and the further property:

(vi) If $X$ is a cylinder set on $R_{\alpha}(\alpha<\beta)$, then so is $\theta(X)$.

To extend the definition of $\theta(X)$ to the cylinder sets on $R_{\beta}$, we consider two cases.

I. If $\beta$ has a predecessor, say $\beta=\gamma+1$, and if a cylinder set $X$ on $R_{\beta}$ is given, we write $\pi_{\beta}(X)=\left(Y^{\prime} \times a_{\gamma}\right) \cup\left(Z^{\prime} \times b_{\gamma}\right)$, where $Y^{\prime}, Z^{\prime}$ are subsets of $R_{\gamma}$ (this determines $Y^{\prime}, Z^{\prime}$ uniquely), and let $Y=Y^{\prime} \times S_{\gamma}$ and $Z=Z^{\prime} \times S_{\gamma}$ be the corresponding cylinder sets on $R_{\gamma}$. Then $\theta(Y)$ and $\theta(Z)$ have already been defined, and we put

$$
\theta(X)=\left\{q \mid q \in \theta(Y) \text { if } q_{\gamma}=a_{\gamma}, q \in \theta(Z) \text { if } q_{\gamma}=b_{\gamma}\right\},
$$

where $q_{\gamma}$ is the $\gamma$ th coordinate of $q \in Q$. It is easy to see that this definition agrees with the previous one if $X$ is a cylinder set on some $R_{\alpha}$ with $\alpha<\beta$ (it suffices to assume $\alpha=\gamma$ ), and that the properties (i) $-($ vi) are preserved.

II. If $\beta$ is a limit ordinal, and $X$ is a cylinder set on $R_{\beta}$, let $\chi_{X}$, or $\chi$ for short, be its characteristic function. For each $\gamma<\beta$ let $T=T_{\beta \gamma}$ denote $\Pi\left\{Q_{\alpha} \mid \gamma \leqq \alpha<\beta\right\}$, and consider the function $f_{X, \gamma}$, or $f_{\gamma}$ for short, defined by

$$
f_{\gamma}(q)=\int_{T} \chi\left(r_{\gamma}, t, s_{\beta}\right) d t
$$

where $r_{\gamma}=\pi_{\gamma}(q)$ and $s_{\beta} \in S_{\beta}$. This function is defined (and between 0 and 1 inclusive) for almost all $q \in Q$; where $f_{\gamma}(q)$ is not defined, we take it to be 0 , say. For each rational number $\rho$, let $G_{\rho}=G_{X, \gamma, \rho}$

${ }^{5}$ If $\beta=\alpha^{*}, \pi_{\beta}$ is of course the identity mapping, and the cylinder sets on $R_{\beta}$ are simply the measurable subsets of $Q$. 
$=\left\{q \mid f_{\gamma}(q)>\rho\right\}$; thus we have $G_{\rho}=Q$ if $\rho<0, G_{1}=\varnothing$, and $G_{\rho} \supset G_{\sigma}$ if $\rho<\sigma ;$ further, $f_{\gamma}(q)=\sup \left\{\rho \mid q \in G_{\rho}\right\}$.

Since $\chi$ is a cylinder function on $R_{\beta}, f_{\gamma}$ is a cylinder function on $R_{\gamma}$, and so $G_{\rho}$ is a cylinder set on $R_{\gamma}$. Hence the sets $H_{\rho}=\theta\left(G_{\rho}\right)$ are defined and have the properties: $H_{\rho}=Q$ if $\rho<0, H_{1}=\varnothing$, and $H_{\rho} \supset H_{\sigma}$ if $\rho<\sigma$. Let $h_{\gamma}(q)=\sup \left\{\rho \mid q \in H_{\rho}\right\}$ (which also equals inf $\left\{\sigma \mid q \notin H_{\sigma}\right\}$ ); then, since $H_{\rho}$ and $G_{\rho}$ differ by at most a null set, we have $h_{\gamma}=f_{\gamma}$ almost everywhere. Let $h(q)=\lim \inf h_{\gamma}(q)$ as $\gamma \rightarrow \beta$. We define

$$
\theta(X)=\{q \mid h(q)=1\},
$$

and show that this leads to the desired properties.

We first verify (iv), which implies that $\theta(X)$ is measurable. By $[3,(5)]$, we can alter $X$ by a null set, replacing $\chi$ by an equivalent function $\chi^{\prime}$, in such a way that the altered $X$ (and so $\chi^{\prime}$ ) depends on only countably many coordinates. The functions $f_{\gamma}$ are replaced by functions $f_{\gamma}^{\prime}$, say, which differ from them only on null sets; hence, for each $\gamma$, the corresponding sets $G_{\rho}, G_{\rho}^{\prime}$ differ only by null sets, and so the sets $I_{\rho}, H_{\rho}^{\prime}$ are the same (exactly). Thus the functions $h_{\gamma}, h_{\gamma}^{\prime}$ are the same. As $\gamma \rightarrow \beta$, we have that $f_{\gamma}^{\prime} \rightarrow \chi^{\prime}$ almost everywhere, by [3], and there are only countably many different functions $f_{\gamma}^{\prime}$ (cf. [3, Case III]). After excluding only countably many null sets, we thus have

$$
h_{\gamma}(q)=h_{\gamma}^{\prime}(q)=f_{\gamma}^{\prime}(q) \rightarrow \chi^{\prime}(q)=\chi(q)
$$

for almost every $q \in Q$. Thus $h=\chi$ almost everywhere, and (iv) follows.

Property (iii), that $\theta(X)$ depends only on the measure class of $X$, is similarly deduced from the fact that altering $G_{\rho}$ by a null set does not alter $H_{\rho}$. The verifications of (i), (v) and (vi) likewise present no difficulties, but that of (ii) is a little more troublesome. If $X$ and $Y$ are cylinder sets on $R_{\beta}$, and $\gamma<\beta$, we have

$$
\int_{T}\left(1-\chi_{X}\right)\left(1-\chi_{Y}\right) d t \geqq 0,
$$

and so $1+f_{X \cap Y, \gamma} \geqq f_{X, \gamma}+f_{Y, \gamma}$, almost everywhere. It follows that, for any rationals $\rho$ and $\sigma, G_{X, \rho} \cap G_{Y, \rho} \subset G_{X \cap Y, \rho+\sigma-1}$ except for a null set, and therefore (from the properties of $\theta$ ) $H_{X, \rho} \cap H_{Y, \sigma} \subset H_{X \cap Y, \rho+\sigma-1}$ (without exception), whence $1+h_{X \cap Y, \gamma}(q) \geqq h_{X, \gamma}(q)+h_{Y, \gamma}(q)$ for all $q \in Q$. On taking the lim inf as $\gamma \rightarrow \beta$, we obtain the desired relation for $\theta$.

Finally, if $X$ is a cylinder set on $R_{\gamma^{\prime}}$ for some $\gamma^{\prime}<\beta$, the transfinite sequence $\left\{f_{\gamma}\right\}$ becomes constant and equal to $\chi$ when $\gamma^{\prime}<\gamma<\beta$, from 
which it is easily seen that $h_{\gamma}=\chi_{\theta(X)}$ for $\gamma^{\prime}<\gamma<\beta$, so that the new definition of $\theta(X)$ is consistent with the old.

Corollary. Theorem 1 applies also to the product $P=\prod\left\{I_{\alpha} \mid \alpha<\alpha^{*}\right\}$ of a well-ordered family of closed unit intervals.

Here the "elementary sets" are taken to be those of the form $\prod\left\{J_{\alpha} \mid \alpha<\alpha^{*}\right\}$ where $J_{\alpha}$ is, for each $\alpha$, one of the binary intervals $\left\{t \mid 0 \leqq t \leqq 1 / 2^{m}\right\}, \quad\left\{t \mid(p-1) / 2^{m}<t \leqq p / 2^{m}\right\}, \quad p=2,3, \cdots, 2^{m}, \quad m$ $=0,1,2, \cdots$, in $I_{\alpha}$, and $J_{\alpha}=I_{\alpha}$ for all but a finite set of $\alpha$ 's.

Proof. Let $t \in I_{\alpha}$ have binary decimal expansion $t=\sum_{1}^{\infty} t_{n} / 2^{n}$ (where $t_{n}=0$ or 1 ; when two expansions are available, the nonterminating one is to be used), and define

$$
g_{\alpha}(t)=\left(t_{1}, t_{2}, \cdots\right) \in \prod Q_{\alpha n} \quad(n=1,2, \cdots) .
$$

Thus $g_{\alpha}$ gives a 1-1 measure-preserving correspondence between $I_{\alpha}$ and $\prod_{n} Q_{\alpha n}-N_{\alpha}$, where $\prod Q_{\alpha n}$ is the product of countably many two-point measure spaces of the previous type, and $N_{\alpha}$ is countable and so null. The factors $Q_{\alpha n}\left(\alpha<\alpha^{*}, n=1,2, \cdots\right)$ are well-ordered in an obvious way, and the product mapping $g=\left\{g_{\alpha} \mid \alpha<\alpha^{*}\right\}$ gives a 1-1 correspondence between $P=\prod I_{\alpha}$ and a certain subset $K$ of $Q=\prod_{\alpha, n} Q_{\alpha n} . K$ need not be measurable, but can be shown (from $[3,(5)])$ to have outer measure 1 . Thus the outer measure on $Q$ provides the well-known relative measure on $K$ (for sets of the form $K \cap Y$ where $Y$ is measurable in $Q$ ); and $g$ will be measure- and measurability-preserving in the sense of this relative measure. ${ }^{6}$ Given a measurable set $X \subset P$, there is a measurable extension $X^{*} \subset Q$ of the relatively measurable set $g(X) \subset K$, and $X^{*}$ is unique to within a null set. Hence $\theta\left(X^{*}\right)$ is determined uniquely, and we define $\theta^{\prime}(X)$ $=g^{-1}\left(\theta\left(X^{*}\right)\right)$. Then $\theta^{\prime}$ has the properties asserted for the lower density set in $P$, because $\theta$ has them in $Q$.

REMARK. A similar theorem and proof apply if, from each interval $I_{\alpha}$, an arbitrary null set (which may vary with $\alpha$ ) is removed.

It is of interest to see what the above definition amounts to in the case of a single unit interval $I$. The imbedding $g$ of $I$ in $\Pi Q_{n}$, $n=1,2, \cdots$, combined with the construction in Theorem 1 , reduces to the following. The set $\theta^{\prime}(X)$ is precisely the set at which the "lower density" of $X$ is 1 , where this "lower density" is obtained as usual except that, instead of using symmetrical intervals $(t-\epsilon, t+\epsilon)$ to calculate it at $t \in I$, we use the half-open binary intervals $\left((p-1) / 2^{m}, p / 2^{m}\right)$ which contain $t$.

${ }^{6} \mathrm{We}$ omit the details; the argument is similar to one occurring at the end of the proof of Theorem 3 below. 


\section{Representative sets.}

THEOREM 2. We can choose, for each class $x$ of measurable sets modulo null sets in $Q\left(=\prod\left\{Q_{\alpha} \mid \alpha<\alpha^{*}\right)\right.$, a representative set $R(x) \subset Q$, in such a way that $R(x)$ is measurable and in the measure class $x$, $R(o)=\varnothing, R(-x)=Q-R(x), R(x \wedge y)=R(x) \cap R(y)$ (and therefore $R(x \vee y)=R(x) \cup R(y)$ ), and further $R\{F\}=F$, where $F$ is any finite union of elementary sets and $\{F\}$ is its measure class.

The sets $R(x)$ are chosen by transfinite induction, using the axiom of choice, in exactly the same way as in [4]. This method guarantees $\theta(X) \subset R(x) \subset Q-\theta(Q-X)$, where $\theta$ is as in Theorem 1 and $X \in x$. If $F$ is a finite union of elementary sets, then so is $Q-F$, so that $\theta(F)=F$ $=Q-\theta(Q-F)$, and hence $R\{F\}=F$. The other properties are proved in $[4]$.

Corollary. Theorem 2 applies also to any product of unit intervals.

THeorem 3. Let $S$ be any measure space, with a complete, $\sigma$-finite, non-negative, countably additive measure $\mu$, and suppose that $\mu(S)>0$. We can choose, for each class $x$ of measurable sets modulo null sets in $S$, a representative set $R(x) \subset S$, in such a way that $R(x)$ is measurable and in the measure class $x, R(o)=\varnothing, R(-x)=S-R(x), R(x \wedge y)$ $=R(x) \cap R(y)$, and therefore $R(x \bigvee y)=R(x) \cup R(y)$.

Let $E$ be the measure algebra of $S$ modulo null sets. There are at most $\boldsymbol{\aleph}_{0}$ atoms $u_{1}, u_{2}, \cdots$, in $E$, and $e-V u_{n}$ (where $e$ is the unit element of $E$ ) can be written as the union of countably many disjoint elements of finite measure, each of which (by [2]) can be written as the union of countably many disjoint homogeneous elements. Thus we have $e-V u_{n}=V v_{m}(m=1,2, \cdots)$ where each principal ideal $E\left(v_{m}\right)$ is nonatomic, homogeneous and of finite positive measure; by [2], $E\left(v_{m}\right)$ is isomorphic (apart from a scale factor) to the measure algebra of a product of intervals or (what is the same thing algebraically) a product $Q^{m}$ of 2-point spaces.

We pick disjoint sets $U_{n} \in u_{n}, V_{m} \in v_{m}$, and note that (because $\mu(S)>0$ ) we may assume $\cup U_{n} \cup \cup V_{m}=S$. It will evidently suffice to choose suitable representatives in the spaces $U_{n}, V_{m}$ separately, for then we have only to define (for any $x \in E) \quad R(x)=\bigcup R\left(x \wedge u_{n}\right.$ ) $\cup \cup R\left(x \wedge v_{m}\right)$.

The representative problem for the atomic space $U_{n}$ is trivial $\left(R\left(u_{n}\right)=U_{n}, R(o)=\varnothing\right)$, so we have only to consider $V_{m}$. That is, the problem is reduced to the case in which $S$ is set-isomorphic to $Q$ $=\prod\left\{Q_{\alpha} \mid \alpha<\alpha^{*}\right\}$. 
Let $\phi$ be a measure-preserving isomorphism between the measure algebras of $Q$ and of $S$. For each $\alpha$, let

$$
A_{\alpha}=\left\{q \mid q \in Q, q_{\alpha}=a_{\alpha}\right\}, \quad B_{\alpha}=\left\{q \mid q \in Q, q_{\alpha}=b_{\alpha}\right\} ;
$$

choose $C_{\alpha} \in \phi\left\{A_{\alpha}\right\}$, and write $D_{\alpha}=S-C_{\alpha}$; thus $D_{\alpha} \in \phi\left\{B_{\alpha}\right\}$, and $\mu\left(C_{\alpha}\right)=1 / 2=\mu\left(D_{\alpha}\right)$. We define a mapping $f$ of $S$ in $Q$ by the rule: $f(p)=\left\{q_{\alpha}\right\}$ where $q_{\alpha}=a_{\alpha}$ if $p \in C_{\alpha}$, and $q_{\alpha}=b_{\alpha}$ if $p \in D_{\alpha}$. Clearly $f^{-1}\left(A_{\alpha}\right)=C_{\alpha}, f^{-1}\left(B_{\alpha}\right)=D_{\alpha}$. It follows that for every elementary set $Y$ of $Q, f^{-1}(Y) \in \phi\{Y\}$, and thence that $f^{-1}(Z) \in \phi\{Z\}$ for every set $Z$ in the Borel field generated by the elementary sets. As every null set $N$ is contained in a null set in this Borel field, we have that $f^{-1}(N)$ is null; and so, finally, $f^{-1}(X) \in \phi\{X\}$ for every measurable $X \subset Q$. By Theorem 2, we can suppose representative sets $R(x)$ chosen for the measure classes of $Q$; now if $y$ is any measure class in $S$, we define $R^{\prime}(y)=f^{-1}\left(R\left(\phi^{-1}(y)\right)\right)$, and readily verify that $R^{\prime}(y) \in y$ and that the sets $R^{\prime}(y)$ provide the desired representatives in $S$.

THEOREM 4. For any system of representative sets as in Theorem 3, all intersections and unions of representative sets are measurable.

Let $\left\{x_{\lambda} \mid \lambda \in \Lambda\right\}$ be any system (not necessarily countable) of elements of $E$, the measure algebra of $S$, and let $V x_{\lambda}=z$. Since $E$ satisfies the countable chain condition, this supremum exists, and there is a countable subfamily $\left\{\lambda_{n}\right\}(n=1,2, \cdots)$ such that $z=\mathrm{V}_{n} x_{\lambda_{n}}$. We have $R\left(x_{\lambda}\right) \subset R(z)$, since $x_{\lambda} \leqq z$, and so $U_{n} R\left(x_{\lambda_{n}}\right) \subset \cup\left\{R\left(x_{\lambda}\right) \mid \lambda \in \Lambda\right\}$ $\subset R(z)$. But $\mathrm{U}_{n} R\left(x_{\lambda_{n}}\right)$ is in the measure class $\mathrm{V}_{n} x_{\lambda_{n}}=z$, and so differs from $R(z)$ by a null set at most. The same is therefore true of $\mathrm{U} R\left(x_{\lambda}\right)$, which (because $\mu$ is complete) must be measurable. The assertion about intersections follows by complementation. Note that we have shown that $U R\left(x_{\lambda}\right)$ (and similarly $\cap R\left(x_{\lambda}\right)$ ) is not only measurable, but in the correct measure class.

CoROllaRY. In every product of two-point factors, or unit intervals, all open sets (in the usual product topology) are measurable.

For every open set is a union of elementary sets; and, by Theorems 2 and 3, all representatives may be chosen so that elementary sets are representatives.

This corollary applies, of course, to products of more general factors; the precise conditions for its validity do not seem easy to determine, but a direct argument shows that the following simple conditions are amply sufficient. Let $S=\prod\left\{S_{\lambda} \mid \lambda \in \Lambda\right\}$ be any product of measure spaces (of measure 1) each of which has a topology with a countable basis of open measurable sets of positive measure. Then 
every open set in $S$ is measurable, in the usual product topology and (completed) product measure.

We remark in conclusion that, by an argument similar to the proof of Theorem 4 , it can be shown that if $\left\{X_{\lambda}\right\}$ is any family of subsets of the line, each having the properties $D^{*}{ }_{X_{\lambda}}(t)>0$ for each $t \in X_{\lambda}$, $D_{* x_{\lambda}}(t)<1$ for each $t \notin X_{\lambda}$, then $U X_{\lambda}$ and $\cap X_{\lambda}$ are measurable, and are in the respective measure classes $\vee\left\{X_{\lambda}\right\}, \Lambda\left\{X_{\lambda}\right\}$. Theorem 4 can be generalised in a similar way.

\section{REFERENCES}

1. J. Dieudonné, On a theorem of Jessen, Fund. Math. vol. 37 (1950) pp. 242-248.

2. D. Maharam, On homogeneous measure algebras, Proc. Nat. Acad. Sci. vol. 28 (1942) pp. 108-111.

3. - On two theorems of Jessen, Proc. Amer. Math. Soc. vol. 9 (1958) pp. 995-999.

4. J. von Neumann, Algebraische Repräsentanten der Funktionen bis auf eine Menge vom Masse Null, J. Reine Angew. Math. vol. 165 (1931) pp. 109-115.

5. J. von Neumann and M. H. Stone, The determination of representative elements in the residual classes of a Boolean algebra, Fund. Math. vol. 25 (1935) pp. 353-378.

The University, Manchester, England 